\title{
$n$
}

NASA Technical Memorandum 102094

\section{Tailoring of Composite Links for Optimal Damped Elasto-Dynamic Performance}

D.A. Saravanos and C.C. Chamis

Lewis Research Center

Cleveland, Ohio

Prepared for the

1989 Design Automotive Conference

sponsored by the American Society of Mechanical Engineers

Montreal, Canada, September 17-20, 1989

\section{N/SA}




\title{
TAILORING OF COMPOSITE LINKS FOR OPTIMAL DAMPED ELASTO-DYNAMIC PERFORMANCE
}

\author{
D. A. Saravanos*, and C. C. Chamis \\ National Aeronautics and Space Administration \\ Lewis Research Center
}

Cleveland, $\mathrm{OH} 44135$

\begin{abstract}
A method is developed for the optimal design of composite links based on dynamic performance criteria directly related to structural modal damping and dynamic stiffness. An integrated mechanics theory correlates structural composite damping to the parameters of basic composite material systems, laminate parameters, link shape, and modal deformations. The inclusion of modal properties allows the selective minimization of vibrations associated with specific modes. Ply angles and fiber volumes are tailored to obtain optimal combinations of damping and stiffness. Applications to simple composite links indicate wide margins for trade-offs and illustrate the importance of various design variables to the optimal design.
\end{abstract}

\section{INTRODUCTION}

Fiber reinforced composites show great potential as candidate materials for high-speed mechanisms and high-speed robotic manipulators. Continuous fiber composites provide superior specific modulus and specific strength allowing the fabrication of stiff, strong, and light-weight links. In this 1w... ner, positioning errors associated with the static or drnamir

* National Research Council - NASA Research Associate 
response are readily decreased, since the elastic deflections are reduced, the strength is increased, and the mass of the links is also decreased resulting in lower inertial loading. Composites also provide the unique capability to tailor the anisotropy of the material to meet specific design requirements. For the case of robotic manipulators, tailoring of the material may result in robotic arms with superior and more uniform properties within their working envelope [1].

In addition to high specific modulus and strength, composite materials will incorporate higher material damping into the structure, since most polymer- matrix composites exhibit higher damping factors by an order of magnitude compared to commonly used metallic alloys. Properly designed composite links can provide higher passive damping, and they may further improve accuracy, strength, and fatigue endurance by attenuating undesirable elasto-dynamic phenomena such as overshooting, long settling times and structural resonances. Recent work $[2,3]$ has shown that structural composite damping is highly tailorable, and is primarily controlled by constituent parameters (fiber/matrix properties, volume fraction), laminate parameters (ply angles/thicknesses, stacking sequence), and the deformation of the articulated structure (mode shapes). The same study has also demonstrated that any increase in damping usually results in decreased stiffness and possibly strength, therefore, any tailoring of the composite material for optimal damped response will be based on trade-offs between damping, stiffness, and strength. In order to realize full benefits from the damping capacity of composite materials, integrated methodologies for the optimal design of composite links should be developed entailing: (1) capability for tailoring the basic composite materials and/or laminate for maximum damping, stiffness, and strength; (2) dynamic design criteria based, if possible, on the performance of the articulated structure; (3) three-dimensional modeling capacity, as the vibrational response of robotic manipulators and mechanisms is in general three-dimensional. The current paper presents the development of such a formal design method allowing the tailoring of composite links for optimal elasto-dynamic performance.

Limited research has been reported on the optimal design of composite links includ- 
ing damping as design criterion. The flexural damping capacity of laminated beams [4] and tubular composite manipulator links $[5,6]$ has been optimized with bounds on design parameters and bending rigidity. Ply angles, ply thicknesses, fiber volume fractions, and cross-sectional dimensions were utilized as design parameters. The present optimal design method is targeted to three-dimensional kinematic configurations. It implements criteria based on global structural dynamic properties rather than on local properties, and it readily incorporates micromechanics for all properties of the composite material, including damping. The method can be used to perform each or combinations of the following functions: (1) prediction of optimal laminate configurations (ply angles and ply thicknesses); (2) optimal tailoring of the composite material in terms of volume fraction, matrix and fiber properties; and (3) prediction of optimal link shapes. The method provides the unique feature to model and optimize the structural damping of individual vibration modes, therefore, superior tailoring of the composite material may result by selective minimization of positioning errors associated with the more predominant modes. In view of the later feature, the design method may be readily combined with active vibration control, such that the elasto-dynamic effects of higher modes are passively minimized by tailoring the composite links, while vibrations from lower modes are actively suppressed.

The performance criteria used in this work are directly related to the damping, static deflections, and dynamic deflections of the composite structure. These properties are modeled using finite element analysis. Composite damping simulation capabilities have been incorporated into a three-dimensional triangular plate element. The optimal design problem is formulated in standard non-linear programming form and is numerically solved with the feasible directions method. For a preliminary assessment regarding the merits of the method, the optimal design of simple links is investigated.

\section{NOMENCLATURE}

$\left[A_{d}\right] \quad 3 \times 3$ Extensional laminate damping matrix.

$\left[A_{s}\right] \quad 3 \times 3$ Extensional laminate stiffness matrix.

$[B] \quad$ Strain shape function matrix. 
$[C],[c] \quad$ Global and modal damping matrices.

$\left[C_{d}\right] \quad 3 \times 3$ Coupling laminate damping matrix.

$\left[C_{s}\right] \quad 3 \times 3$ Coupling laminate stiffness matrix.

$\left[D_{d}\right] \quad 3 \times 3$ Flexural laminate damping matrix.

$\left[D_{s}\right] \quad 3 \times 3$ Flexural laminate stiffness matrix.

E Normal modulus.

$\left[E_{l}\right],\left[E_{c}\right] \quad$ On-axis and off-axis ply stiffness matrices.

$G \quad$ Shear modulus.

$k \quad$ Volume ratio.

$\left[K^{*}\right],[k] \quad$ Global and modal stiffness matrices.

$[M],[m] \quad$ Global and modal mass matrices.

$N_{L} \quad$ Number of plies in a laminate.

$q \quad$ Modal vector.

$t \quad$ Time.

$t_{l} \quad$ Ply thickness.

u† Displacement vector.

$U^{d} \quad$ Undamped dynamic amplitude.

$u_{x}, u_{y}, u_{z} \quad$ Deflections along $\mathrm{x}, \mathrm{y}, \mathrm{z}$ structural axes.

$w, \delta w \quad$ Stored and dissipated specific strain energies.

$W, \delta W \quad$ Stored and dissipated strain energies.

$z \quad$ Distance from laminate mid-surface.

$\epsilon \quad$ Engineering strain.

$\theta \quad$ Fiber orientation angle.

$\kappa \quad$ Curvature.

$\nu \quad$ Poisson's ratio.

$\sigma \quad$ Stress.

$\psi \quad$ Specific damping capacity.

$\dagger$ Bold characters indicate vectors 


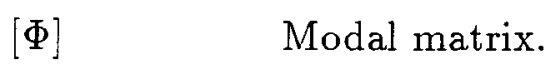

$\phi_{\mathbf{x}}, \phi_{\mathbf{y}}, \phi_{\mathbf{z}} \quad$ Rotations about $\mathrm{x}, \mathrm{y}, \mathrm{z}$ structural axes.

$\omega$

Angular velocity.

\section{Subscripts}

$b$

$\mathcal{c}$

$\epsilon$

$f$

i

$l$

$L$

$m$

$n$

$r p$

$t$

\section{Superscripts}

$d$

$L$

$o$

$s$

$U$

\section{Direction}

11

22

12

$x x$

yy
Ply bottom.

Ply (off-axis).

Finite element.

Fiber.

Node.

Ply (on-axis).

Laminate.

Matrix.

$\mathrm{n}$-th mode.

Ply interface.

Ply top.

Dynamic.

Lower.

Laminate mid-surface.

Static.

Upper.

Normal longitudinal.

Normal in-plane transverse.

Shear in-plane.

In-plane normal, off-axis, $\mathrm{x}$-direction.

In-plane normal, off-axis, y-direction. 
In-plane shear, off-axis, $y$-direction.

\section{COMPOSITE STRUCTURAL DAMPING}

In this section an integrated theory for modeling structural composite damping is described. Analogous theories were utilized for other mechanical properties [7]. The damping of the composite link is simulated based on an upward synthesis scheme. First, the on-axis damping of each composite material (damping along the material axes) is synthesized from the constituent fiber/matrix properties and volume fractions. Then, the damping capacities of plies subjected to off-axis loading (off-axis plies) are calculated, and subsequently the local laminate damping capacity is formulated. Integration of local laminate damping over the whole structure provides the global structural damping capacity at a particular deformation state. Finite element discretization is utilized for the numerical integration of local laminate damping. The same finite element mesh is also utilized to simulate the static and dynamic response.

\section{On-Axis Composite Damping}

A simplified micromechanics theory [2] has been utilized for the synthesis of hysteretic on-axis composite damping. The micromechanics equations for on-axis damping are presented in the Appendix. The specific strain energy released per cycle due to in-plane on-axis cyclic loading is:

$$
\delta w_{l}=\frac{1}{2}\left\{\sigma_{l}\right\}^{T}\left[\psi_{l}\right]\left\{\epsilon_{l}\right\}
$$

\section{Off-Axis Composite Damping}

Off-axis composite specific damping capacities (SDC's) are synthesized from the onaxis properties and the ply angle. The transformation law is given in the Appendix. The off-axis damping matrix $\left[\psi_{c}\right]$ is fully populated due to in-plane coupling between extension and shear. As has been demonstrated in ref. [2] coupling provides an additional physical mechanism for strain energy dissipation, and consequently, higher damping. The specific strain energy released per cycle during cyclic in-plane off-axis loading is,

$$
\delta w_{c}=\frac{1}{2}\left\{\sigma_{c}\right\}^{T}\left[\psi_{c}\right]\left\{\epsilon_{c}\right\}
$$




\section{Laminate Damping}

The laminate damping is ensembled from the damping contributions of each off-axis ply. As shown in the Appendix and ref. [3], the damping capacity of a general composite laminate is formally described by three $3 \times 3$ fully-populated matrices: (1) the extensional damping matrix $\left[A_{d}\right]$ associated with extensional deformations only; (2) the flexural damping matrix $\left[D_{d}\right]$ associated with bending and torsional deformations; and (3) the extension-flexure coupling damping matrix $\left[C_{d}\right]$ associated with coupled extensionflexure deformations. The coupling damping matrix $\left[C_{d}\right]$ is identically zero for symmetric laminates. In addition to hysteretic ply damping, the laminate damping includes also contributions from hysteretic interlaminar strain energy dissipated in the interply matrix layer, as a result of the interlaminar shear stress developed between two adjacent plies in order to restrict their relative local movement (scissors effect). The dissipated strain energy through-the- thickness of the laminate per unit area per cycle is:

$$
\delta W_{L}=\frac{1}{2}\left\{\epsilon^{\mathbf{o}} \quad \kappa\right\}\left[\begin{array}{ll}
{\left[A_{d}\right]} & {\left[C_{d}\right]} \\
{\left[C_{d}\right]} & {\left[D_{d}\right]}
\end{array}\right]\left\{\begin{array}{c}
\epsilon^{\mathbf{o}} \\
\kappa
\end{array}\right\}
$$

As seen in eq. (3), the laminate damping is a local structural property, depending on the local deformation, the basic constituent composite materials, and laminate configuration. Correlation with experimental measurements has yielded excellent agreement [3].

\section{Structural Modal Damping}

The local laminate damping provides rough estimates regarding the global damping capacity of simple structural components and loading conditions where the local strain and curvature remain mostly uniform over the structure. However, most composite structures of practical interest would exhibit more complicated and coupled deformations, and their global damping capacity would be the integrated contribution of local damping over the whole volume at a particular deformation shape.

Since the elasto-dynamic response of any structure is a linear combination of individual vibration modes, structural damping associated with each mode would provide a natural and meaningful measure regarding the overall damping capacity of the composite structure. 
In the remaining sections, the global damping capacity of the structure for a particular mode shape will be identified as structural modal damping. In this manner, an array of modal damping factors is generated, each one representing the damping capacity of the structure vibrating solely at the respective mode shape. The modal specific damping capacity (SDC) associated to the $n$-th vibration mode would be:

$$
\psi_{n}=\frac{\int_{V} \delta w_{n} d V}{\int_{V} w_{n} d V}
$$

where $\delta w_{n}$ and $w_{n}$ represent the dissipated and stored specific strain energies per cycle. Utilizing eq. (3), the modal damping of plate/shell composite structures becomes,

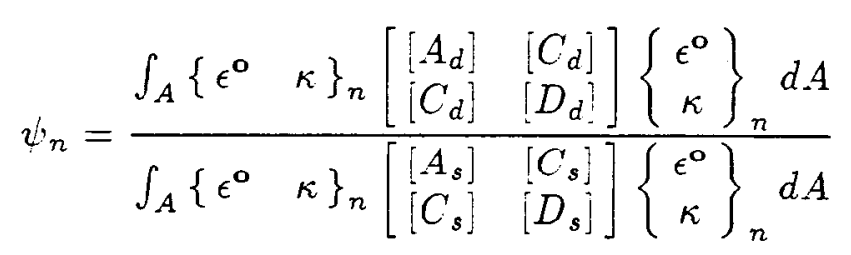

The integrations in eq. (5) are performed numerically using finite element discretization. Such a finite element scheme has been developed for an isoparametric triangular plate element with 3 nodes, 6 degrees of freedom per node $u_{i}=\left\{u_{x}, u_{y}, u_{z}, \phi_{x}, \phi_{y}, \phi_{z}\right\}$, linear shape functions for membrane deflections, and cubic shape functions for flexure.

Integration is first performed over the area of the triangular finite element. Given the shape functions for membrane and flexural deflections, the mid-plane strain and curvature at a point is related to the nodal displacement vector,

$$
\left\{\begin{array}{c}
\epsilon^{\mathbf{o}} \\
\kappa
\end{array}\right\}=[B] \mathbf{u}_{\mathbf{e}}
$$

Based on this relation, the damping and strain energies per cycle, over the area of the triangular element would be respectively:

$$
\begin{gathered}
\delta W_{e, n}=\int_{A_{\mathbf{e}}} \mathbf{u}_{\mathbf{e}, \mathbf{n}}{ }^{T}[B]^{T}\left[\begin{array}{ll}
{\left[A_{d}\right]} & {\left[C_{d}\right]} \\
{\left[C_{d}\right]} & {\left[D_{d}\right]}
\end{array}\right][B] \mathrm{u}_{\mathbf{e}, \mathbf{n}} d A_{e} \\
W_{e, n}=\int_{A_{e}} \mathbf{u}_{\mathbf{e}, \mathbf{n}}{ }^{T}[B]^{T}\left[\begin{array}{ll}
{\left[A_{s}\right]} & {\left[C_{s}\right]} \\
{\left[C_{s}\right]} & {\left[D_{s}\right]}
\end{array}\right][B] \mathrm{u}_{\mathbf{e}, \mathbf{n}} d A_{e}
\end{gathered}
$$


Both integrals are calculated numerically using three-point quadrature. Once the dissipated and stored strain energies are calculated for each element, then their summation provides the total dissipated and stored energies. The modal SDC of the $\mathrm{n}$-th node $\psi_{n}$ is then assembled according to equation (5).

$$
\psi_{n}=\frac{\sum_{j=1}^{N_{e}}\left(\delta W_{e, n}\right)_{j}}{\sum_{j=1}^{N_{e}}\left(W_{e, n}\right)_{j}}
$$

\section{ELASTO-DYNAMIC RESPONSE}

The dynamic elastic response of composite links is simulated with finite element analysis. The plate triangular element mentioned previously is used for this purpose. This type of element was preferred in this initial study, because it provides more accurate modeling and insight, than the beam finite elements used in previous studies [1,4-6]. In this manner, a reliable data-base of results is assembled for future assessments of the accuracy of less expensive beam elements. Using finite element discretization, the elasto-dynamic response of the composite link may be simulated by the following system of $n$ discrete dynamic equations,

$$
[M]\{\ddot{u}\}+[C]\{\dot{u}\}+[K]\{u\}=\{F(t)\}
$$

The damping matrix is formulated in the modal space. A typical exciting force for composite robotic manipulators would involve: surface tractions $F_{s}(t)$, body forces $F_{b}(t)$, inertial forces due to local, centrifugal and Coriolis acceleration $F_{i}(\ddot{r})$, and hygrothermal forces $F_{H T}(t)$.

$$
\{F(t)\}=\left\{F_{s}(t)\right\}+\left\{F_{b}(t)\right\}+\left\{F_{r}(\ddot{r})\right\}+\left\{F_{H T}(t)\right\}
$$

This study neglects the effects of body and hygrothermal forces, hence, is limited to links operating in room temperature and dry environment. Hygrothermal effects may be significant in the design of composite links, and the method outlined here has provisions to model them, however, the design problem is complicated beyond the scope of the current 
paper, as the damping and stiffness of the composite depend also on temperature and moisture variations [3].

The dynamic system in equation (9) is transformed to the $m \times m$ modal space through the linear transformation [8]:

$$
\{u\}=[\Phi]\{q\}
$$

where $q$ is the modal displacement vector, and $[\Phi]$ contains the first $m$ normalized undamped eigenvectors. For proportional damping, the transformation yields the reduced uncoupled dynamic system,

$$
[m]\{\ddot{q}\}+[c]\{\dot{q}\}+[k]\{q\}=\{\bar{f}(t)\}
$$

where,

$$
\begin{gathered}
{\left[m_{]}=[\Phi]^{T}[M][\Phi]\right.} \\
{[c]=[\Phi]^{T}[C][\Phi]} \\
{[k]=[\Phi]^{T}[K][\Phi]} \\
\{\bar{f}(t)\}=[\Phi]^{T}\{F(t)\}
\end{gathered}
$$

The decoupled damping matrix is formulated from the first m modal SDC's from equations (5-8). For general load histories, the differential equations are numerically solved.

\section{Impulse Response}

The response of the composite link to an impact force $\{F\} h(t)$, where $h(t)$ is the unit impulse, would be [8]:

$$
u_{j}(t)=\sum_{n=1}^{m} \Phi_{j n} \bar{f}_{n} \frac{e^{-\zeta_{n} \omega_{n} t}}{m_{n} \omega_{d, n}} \sin \left(\omega_{d, n} t\right)
$$

where $\omega_{d, n}$ is the damped natural angular velocity of the $\mathrm{n}$-th mode, $\mathrm{j}$ any nodal displacement of the dynamic system (9), and $\{\bar{f}\} h(t)=[\Phi]^{T}\{F\} h(t)$. Eq. (13) is rewritten in the form:

$$
u_{j}(t)=\sum_{n=1}^{m} U_{j, n}^{d} \frac{e^{-\zeta_{n} \omega_{n} t}}{\sqrt{1-\zeta_{n}^{2}}} \sin \left(\omega_{d, n} t\right)
$$


Eq. (14) illustrates the effect of modal undamped amplitudes $U_{j, n}^{d}$ and modal damping ratios on the impulse response. The undamped amplitudes of each mode,

$$
U_{j, n}^{d}=\frac{\Phi_{j n} \bar{f}_{n}}{m_{n} \omega_{n}}
$$

are used as performance measures for the dynamic stiffness of composite links in the following section.

\section{OPTIMAL DESIGN METHOD}

Most common transient vibration problems in high-speed manipulation are usually induced by impulsive excitation. During minimal time (bang-bang) control, the actuators are mostly operating at their maximum torque capacities. The minimal time movement of the end-effector between two points typically involves an acceleration phase under maximum positive torque, immediately followed by a deceleration phase under maximum negative torque. More complicated maneuvers of articulated robotic arms with multiple degrees of freedom exhibit the same fundamental pattern of switchings between acceleration and deceleration. Impact excitations are applied during the initiation of the motion, each switching from acceleration to deceleration and at the end of the rigid body motion.

It is reasonable, therefore, to tailor the composite links for optimal elasto-dynamic response in impulsive excitation. As seen in eq. (14), a feasible way to control the elastodynamic positioning error would be by restraining each undamped modal amplitude within acceptable limits. One advantage of using dynamic amplitudes, in addition to static stiffness, is that the modal mass is also introduced into the design. In this manner, the elasto-dynamic vibration will remain within acceptable bounds, while the maximization of modal damping will ensure minimal settling times. The static stiffness is also bounded, such that, acceptable static deflections under the maximum static loads are ensured.

Two different optimal design cases are formulated, each one representing different design considerations and requirements. Both cases are presented in standard non-linear programming form.

\section{Case 1:}


The present design case aims to maximize the damping capacity of the first mode, in conjunction with other constraints. The first mode has the lower frequency and requires the higher settling time. Preliminary investigations regarding the vibration of off-axis composite beams have shown that the first mode is a bending mode. Case (1) is most suitable when active control is infeasible or undesirable, and the only remaining option is passive vibration control. In this context, the optimal design problem is formulated as follows:

$$
\max \left(\psi_{1}\right)
$$

subjected to:

( i.) Upper and lower bounds on design variables:

$$
\begin{gathered}
-90.0^{\circ} \leq \theta_{i} \leq 90.0^{\circ} \\
0.0 \leq k_{f, i} \leq 0.70
\end{gathered}
$$

where $\theta_{i}$ and $k_{f, i}$ are respectively the ply angle and the fiber volume ratio (FVR) of the $\mathrm{i}$-th sublaminate.

( ii.) Constraints on $q_{s}$ static displacements at the tip of the link:

$$
u_{j}^{s} \leq U_{j}^{s, U} \quad j=1, \ldots, q_{s}
$$

( iii.) Constraints on $m$ undamped modal dynamic amplitudes as provided by eq. (15) for $q_{d}$ displacements at the tip of the link:

$$
U_{j, n}^{d} \leq U_{j, n}^{d, U} \quad j=1, \ldots, q_{d}, \text { and } n=1, \ldots, m
$$

Case 2:

This case is suitable for manipulators with active vibration controllers. Active controllers are most effective up to a frequency limit, therefore, they successfully suppress the static deflections and the elasto-dynamic deflections from the first vibration modes. Passive attenuation of higher modes is then desirable. The present methodology provides the capability to design composite links such that the passive damping of higher modes is maximized and the respective undamped dynamic amplitudes remain within acceptable limits. 
Assuming that passive vibration control is desirable for modes $m_{1}$ to $m$, the following proposed performance criteria will maximize the modal damping of the mode with the higher dynamic amplitude:

$$
\max \left(\psi_{n}\right), \quad m_{1} \leq n \leq m
$$

where the mode $\mathrm{n}$ is selected such that:

$$
U_{n}^{d}=\max \left\{U_{j, m_{1}}^{d}, \ldots, U_{j, m}^{d} ; \quad j=1, \ldots, q_{d}\right\}
$$

subjected to:

( i.) Upper and lower bounds on design variables:

$$
\begin{gathered}
-90.0^{\circ} \leq \theta_{i} \leq 90.0^{\circ} \\
0.0 \leq k_{f, i} \leq 0.70
\end{gathered}
$$

( ii.) Constraints on undamped modal dynamic amplitudes for $q_{d}$ displacements at the tip of the link and for modes $m_{1}$ to $m$ :

$$
U_{j, n}^{d} \leq U_{j, n}^{d, U} \quad j=1, \ldots, q_{d}, \text { and } n=m_{1}, \ldots, m
$$

\section{Design Vector}

In both cases the design vector includes: (1) the ply orientation angle $\theta_{i}$ of each sublaminate (group of $\pm \theta_{i}$ plies), and (2) the fiber volume ratios (FVR's) $k_{f, i}$ of each sublaminate. By considering only composite material parameters, the current study is mainly focused on the tailoring of composite links. The effect of shape optimization and its interaction with material tailoring will be addressed in a future study.

\section{Optimization Algorithm}


Both design cases have been formulated in non-linear programming form and are numerically solved with the method of feasible directions. The feasible directions algorithm is a primal optimization method, and performs a direct search of the feasible design space based on first order sensitivity for objective function and constraints. Primal methods are more suitable for designs involving computationally expensive constraints, like the present one, since they typically require fewer constraint evaluations than the penalty transformation methods. The feasible directions method used in this study incorporated active constraint set and line-search strategies for improved efficiency.

\section{CASE STUDIES}

\section{Assumptions}

Application studies were performed regarding the optimal tailoring of a plate composite link 6 in long, 1 in wide, and 0.2 in thick. This simple composite link was selected in order to obtain an initial assessment of the merits of the present design methodology and to identify the important design parameters. A finite element mesh of 55 nodes and 80 plate triangular elements was used as shown in Figure 1. The link was assumed clamped at one end (nodes 1 to 5 ) and free at the other (nodes 51 to 55). The composite material was high-modulus treated graphite (HM-S) fiber in an epoxy matrix. The mechanical properties of the matrix and the fiber are shown in Table 1 . The material system was selected to match the $50 \%$ FVR HM-S/DX210 graphite/epoxy composite for which experimental damping measurements are provided [9]. The damping properties of the fiber and matrix for this material system were back-calculated from the reported on-axis damping values of the 50\% FVR graphite/epoxy composite (see Table 1) based on the micromechanics equations in the Appendix.

The basic laminate configurations are shown in Figure 2. Both configurations are symmetric and consist of regular angle-ply sublaminates $\left(\theta_{k} /-\theta_{k} / \theta_{k} \ldots\right)$. Configuration (a) involves one sublaminate, and configuration (b) three. Regardless the number of sublaminates, the link consists of 20 plies through the thickness, each 0.01 in thick, such that each ply may be manufactured from 0.005 in thick unidirectional preimpregnated tape. Each 
sublaminate may have a different FVR, consequently, the $\mathrm{i}$-th sublaminate will introduce 2 design variables into the global design vector: the ply angle $\theta_{i}$, and the FVR $k_{f, i}$.

The static load is a uniform transverse (z-axis) force of $5.0 \mathrm{lb} /$ in applied at the tip of the link (nodes 51-55), and the dynamic load a uniform transverse (z-axis) impulse of 0.1 $\mathrm{lb} /$ in applied at the same location. The reference design was taken to be a unidirectional composite link of 50\% HM-S/epoxy, since a unidirectional composite provides very high rigidity but low damping in bending. Unless otherwise stated, the static (z-axis) deflections along the tip were restricted to be less that 0.05 in (2.5 times the maximum static deflection of the unidirectional link), and each individual modal amplitude of the transient (z-axis) response at the middle of the tip (node 53) to be less than 1.87 in (2.0 times the maximum amplitude for the unidirectional link).

\section{Case 1}

Three different subcase studies were performed for this type of problem for: (a) one sublaminate with fixed $50 \%$ FVR, (b) three sublaminates of $50 \%$ FVR, and (c) three sublaminates with varying FVR's.

Subcase 1a. The current link was assumed to consist of one sublaminate with fixed fiber volume ratio. This is the simplest tailoring that can be performed on the composite link, and the design vector involves only the ply angle $\theta_{1}$. The optimal design is shown in Table 2. The optimal, $\pm 27^{\circ}$ angle-ply symmetric laminate provides 4.5 times higher damping for the fundamental mode with respect to the reference unidirectional link and 4.2 times higher damping for the second bending mode. The trade-offs for these damping improvements are higher static compliance by 2.5 times, higher dynamic amplitudes by 1.5 times and lower natural frequencies.

Subcase 1b. The current subcase involves three sublaminates of the same basic material system. Sublaminates 1 and 2 have 4 plies, and sublaminate 3 has 2 plies. The design vector now involves three ply angles. The optimal design is shown in Table 2 . The utilization of three sublaminates has not improved the optimal damping capacity of the fundamental mode compared to the optimal design of Subcase 1a. The resultant optimal 
ply angles demonstrate that the outer sublaminates are critical to the damping and stiffness of the composite link, while the inner sublaminate had a rather insignificant effect. Such type of arrangement was indeed expected, because the first bending mode controls the design. However, the $\pm 5^{\circ}$ plies in sublaminate 3 provide higher in-plane stiffness in $\mathrm{x}$ direction than the optimal design of Subcase 1a. The effect of higher in-plane stiffness is indicated by the higher value of the second natural frequency.

Subcase 1c. In this subcase, the fiber volume ratio of each sublaminate system is incorporated into the design vector, which now consists of 3 ply angles and 3 fiber volume ratios. As seen in Table 2, the introduction of FVR's as design variables has produced a sandwich type of laminate, in that, the fiber volume ratio of the outer sublaminate has reached its upper bound. The outer sublaminate provides more bending stiffness but less damping. The FVR's of the other two sublaminates have been slightly changed. This laminate arrangement has produced almost $8 \%$ higher SDC for the first modal damping and higher SDC for the second bending mode, without any additional penalties on the static stiffness, dynamic amplitudes, and natural frequencies.

All subcases demonstrate wide margins for trade-offs between various modal damping capacities, static and dynamic stiffness, and natural frequencies. Additional insight into the dynamics of the problem is provided by Figures 3 and 4, which illustrate the mode shapes for the unidirectional reference design and the optimum design of Subcase 1c. The bending-torsion coupling is minimal. The optimum design has also switched the order of modes and has affected the SDC's of the torsional modes. Torsional modes may also induce elasto-dynamic deflections at the end-effector of robotic manipulators. Although the current applications are restricted to transverse bending deflections, the torsional deflections may be significant for manipulators with multiple links.

\section{Case 2}

A link with the previous laminate configuration of three sublaminates and three different material systems was optimized. The basic assumption of the current case is that the fundamental mode will be eliminated with active vibration control, hence, the optimum 
tailoring is now solely focused on modes 2 to 5 . In this context, the damping capacity of the most predominant mode between modes 2 and 5 was maximized. The imposed upper bound on the modal dynamic amplitudes of modes 2 to 5 was 0.29 in (2 times the maximum modal amplitude for modes 2 to 5 of the unidirectional link). The optimum design is shown in Table 3. The mode with the higher undamped amplitude is mode 3 (2nd bending), while at the reference design the most predominant mode was mode 4 (2nd bending). The respective modal damping has increased by a factor of 5 , while the respective modal amplitude has been increased by a factor of 2 . The damping capacity of the fundamental mode 1 (1st bending) has been also drastically increased, although was not incorporated into the design criteria. The current case illustrates that the utilization of active controllers in composite robotic manipulators, will not only eliminate the elastodynamic effects of the lower modes, but it will also facilitate the design of composite links with better passive vibration suppression for the higher modes.

The transient responses of the unidirectional and optimum (subcase 1c) designs are shown in Figure 5. Each graph presents the tip vibration (node 53) of the respective design induced by the $z$-axis impulsive load of $0.1 \mathrm{lb} /$ in applied at the tip of the link. The superiority of the optimum design is clearly illustrated. Initially, the dynamic deflections of the optimum design were higher than the dynamic deflections of the unidirectional design, however, because of the higher damping the deflections of the optimum design were quickly reduced to lower values compared to the unidirectional design. At 0.6 secs, the deflections of the optimum design were virtually reduced to $40 \%$ of the respective deflections of the unidirectional link.

\section{SUMMARY}

A formal design method was presented which automates the design of composite links for optimal elasto-dynamic response. The method was based on static and dynamic performance criteria. The proposed dynamic design criteria were directly related to the damping and amplitudes of selected vibration modes. The elasto-dynamic response was simulated with finite element analysis. Simplified, design oriented micromechanics and 
laminate mechanics models for composite damping correlated the performance criteria with laminate and composite material parameters. The present method can be utilized for basic material, laminate, and structural shape optimization. Two distinct design problems were formulated aiming to manipulators with and without active controllers.

The applications illustrate the effect of ply angles, multiple angle-ply sublaminates, and fiber volume fractions on the optimal design. Based on the case studies, the following conclusions are summarized:

1. The resultant optimal laminate configurations and composite material systems significantly improved the damping of bending modes by factors of 5 with respect to the reference unidirectional design, while the undamped dynamic amplitudes only degraded by factors of 1.5. Wide margins for trade-offs exist between damping, and static/dynamic stiffness. These trade-offs can be achieved without changing the geometric shape of the links, and consequently, their mass.

2. Ply angles were the most critical design parameters.

3. Laminates with single or multiple angle-ply sublaminates produced optimal designs of comparable performance.

4. The optimization of fiber volume fractions has produced small improvements in the damping of bending modes.

5. Dynamic properties associated with torsional modes where also found sensitive to variations of laminate and composite material parameters. The actual effect of torsional modes on the end-effector deflections is yet unclear. Minimization of the torsional dynamic effects will be better accomplished with design criteria based on the elasto-dynamic response of the articulated robotic arm, rather than on individual links. 


\section{REFERENCES}

1. Saravanos D.A., 1988, "Structural Optimization of Robotic Manipulators with Fiber Reinforced Composite Materials," Ph.D Thesis, The Pennsylvania State University.

2. Saravanos D.A., and Chamis C.C., 1989, "Unified Micromechanics of Damping for Composite Plies," Journal of Composites Technology and Research, in review.

3. Saravanos D.A., and Chamis C.C., 1989, "Mechanics of Damping for Fiber Composite Laminates Including Hygro-Thermal Effects,' 30th Structures, Structural Dynamics, and Materials Conference, Part 1, AIAA, New York, pp. 296-303.

4. Liao, D.X., Sung, C.K., and Thompson, B.S., 1986, "The Optimal Design of Laminated Beams Considering Damping,' Journal of Composite Materials, Vol. 20, No. 5, pp. 485-501.

5. Sung, C.K., and Thompson, B.S., 1986, “A Methodology for Synthesizing High-Performance Robots Fabricated with Optimally Tailored Composite Laminates, " Journal of Mechanisms, Transmissions, and Automation in Design, Vol. 109, No. 1, pp. 74-86.

6. Liao, D.X., Sung, C.K., and Thompson, B.S., .1987, “The Design of Flexible Robotic Manipulators with Optimal Arm Geometries Fabricated from Composite Laminates with Optimal Material Properties," The International Journal of Robotics Research, Vol. 6, No. 3, pp. 116-130.

7. Murthy, P.L.N., and Chamis, C.C., 1984, "ICAN: Integrated Composites Analyzer," $A I A A$ Paper 84-0974.

8. Meirovitch, L., 1975, Elements of Vibration Analysis, McGraw-Hill, New YorK.

9. Ni, R.G., and Adams, R.D., 1984, “The Damping and Dynamic Moduli of Symmetric Laminated Composite Beams-Theoretical and Experimental Results,' Journal of Composite Materials, Vol. 18, No. 2, pp. 104-121. 


\section{APPENDIX}

The present Appendix summarizes the micromechanics and laminate mechanics theories for composite damping implemented in this work [2,3]. Analogous micromechanics and laminate mechanics theories [7] have been utilized for the remaining mechanical properties of polymer matrix composites.

\section{On-axis Composite damping:}

Longitudinal normal ply SDC $\psi_{l 11}$ :

$$
\psi_{l 11}=\psi_{f 11} k_{f} \frac{E_{f 11}}{E_{l 11}}+\psi_{m n} k_{f} \frac{E_{m}}{E_{l 11}}
$$

Transverse normal ply SDC's $\psi_{122}$ :

$$
\psi_{l 22}=\psi_{f 22} \sqrt{k_{f}} \frac{E_{22}}{E_{f 22}}+\psi_{m n}\left(1-\sqrt{k_{f}}\right) \frac{E_{22}}{E_{m}}
$$

where,

$$
E_{22}=\left(1-\sqrt{k_{f}}\right) E_{m}+\frac{\sqrt{k_{f}} E_{m}}{1-\sqrt{k_{f}}\left(1-\frac{E_{m}}{E_{f 22}}\right)}
$$

In-plane shear ply SDC $\psi_{l 12}$ :

$$
\psi_{l 12}=\psi_{f 12} \sqrt{k_{f}} \frac{G_{12}}{G_{f 12}}+\psi_{m s}\left(1-\sqrt{k_{f}}\right) \frac{G_{12}}{G_{m}}
$$

where,

$$
G_{12}=\left(1-\sqrt{k_{f}}\right) G_{m}+\frac{\sqrt{k_{f}} G_{m}}{1-\sqrt{k_{f}}\left(1-\frac{G_{m}}{G_{f 12}}\right)}
$$

Off-Axis Composite Damping:

$$
\left[\psi_{c}\right]=\left[R_{\sigma}\right]^{T}\left[\psi_{l}\right]\left[R_{\sigma}^{-1}\right]^{T}
$$

where,

$$
\left[R_{\sigma}\right]=\left[\begin{array}{ccc}
\cos ^{2} \theta & \sin ^{2} \theta & \sin 2 \theta \\
\sin ^{2} \theta & \cos ^{2} \theta & -\sin 2 \theta \\
-0.5 \sin 2 \theta & 0.5 \sin 2 \theta & \cos 2 \theta
\end{array}\right]
$$




$$
\begin{aligned}
& {\left[\psi_{l}\right]=\left[\begin{array}{ccc}
\psi_{l 11} & 0 & 0 \\
0 & \psi_{l 22} & 0 \\
0 & 0 & \psi_{l 12}
\end{array}\right]} \\
& {\left[\psi_{c}\right]=\left[\begin{array}{lll}
\psi_{c x x} & \psi_{c x y} & \psi_{c x s} \\
\psi_{c y x} & \psi_{c y y} & \psi_{c y s} \\
\psi_{c s x} & \psi_{c s y} & \psi_{c s s}
\end{array}\right]}
\end{aligned}
$$

\section{Laminate Damping:}

The SDC is expressed in the form of extensional, coupling, and flexural $3 \times 3$ damping matrices.

$$
\begin{gathered}
{\left[A_{d}\right]=\sum_{i=1}^{N_{L}}\left(z_{t}-z_{b}\right)_{i}\left[E_{c}\right]_{i}\left[\psi_{c}\right]_{i}+\sum_{i=1}^{N_{L}-1} H_{i}[S]_{i}\left[\psi_{m}\right]_{i}} \\
{\left[C_{d}\right]=\sum_{i=1}^{N_{L}} \frac{1}{2}\left(z_{t}^{2}-z_{b}^{2}\right)_{i}\left[E_{c}\right]_{i}\left[\psi_{c}\right]_{i}+\sum_{i=1}^{N_{L}-1} z_{r p, i} H_{i}[S]_{i}\left[\psi_{m}\right]_{i}} \\
{\left[D_{d}\right]=\sum_{i=1}^{N_{L}} \frac{1}{3}\left(z_{t}^{3}-z_{b}^{3}\right)_{i}\left[E_{c}\right]_{i}\left[\psi_{c}\right]_{i}+\sum_{i=1}^{N_{L}-1} z_{r p, i}^{2} H_{i}[S]_{i}\left[\psi_{m}\right]_{i}}
\end{gathered}
$$

$\left[\psi_{m}\right]$ is the matrix damping, $H_{j}$ the interply distortion energy coefficient $[7]$, and $\left[E_{c}\right]$ the off-axis ply stiffness matrix,

$$
\begin{gathered}
{\left[E_{c}\right]^{-1}=\left[R_{\sigma}\right]^{T}\left[E_{l}\right]^{-1}\left[R_{\sigma}\right]} \\
{\left[E_{l}\right]^{-1}=\left[\begin{array}{ccc}
\frac{1}{E_{l_{12}}} & -\frac{\nu_{l 21}}{E_{l 22}} & 0 \\
-\frac{\nu_{21}}{E_{112}} & \frac{1}{E_{l 22}} & 0 \\
0 & 0 & \frac{1}{G_{l 12}}
\end{array}\right]}
\end{gathered}
$$


Table 1 Mechanical properties of the HM-S/epoxy system.

\begin{tabular}{lll} 
Epoxy & HM-S Graphite & $50 \%$ HM-S/Epoxy \\
\hline$E_{m}=0.500 \mathrm{msi}$ & $E_{f 11}=55.0 \mathrm{msi}$ & $\psi_{l 11}=0.45 \%$ \\
$G_{m}=0.185 \mathrm{msi}$ & $E_{f 22}=0.9 \mathrm{msi}$ & $\psi_{l 22}=4.22 \%$ \\
$\psi_{m n}=10.30 \%$ & $G_{f 12}=1.1 \mathrm{msi}$ & $\psi_{l 12}=7.05 \%$ \\
$\psi_{m s}=11.75 \%$ & $\nu_{f 12}=0.20$ & \\
& $\psi_{f 11}=0.4 \%$ & \\
& $\psi_{f 22}=0.4 \%$ & \\
& $\psi_{f 12}=0.4 \%$ &
\end{tabular}


Table 2 Optimum designs for Case 1.

\begin{tabular}{|c|c|c|c|c|}
\hline Property & Unidirectional & $\begin{array}{l}\text { Optimum } \\
\text { (Case 1a) }\end{array}$ & $\begin{array}{l}\text { Optimum } \\
\text { (Case 1b) }\end{array}$ & Optimum \\
\hline
\end{tabular}

Ply Angles, degrees

$\begin{array}{llccc}\theta_{1} & 0.0 & 2 \tau .00 & 2 \tau .16 & 30.24 \\ \theta_{2} & 0.0 & - & 2 \tau .30 & 30.49 \\ \theta_{3} & 0.0 & - & 5.21 & 29 . i 6\end{array}$

Fiber volume ratios

$\begin{array}{lllll}k_{f 1} & 0.50 & - & - & 0.69 \\ k_{f 2} & 0.50 & - & - & 0.53 \\ k_{f 3} & 0.50 & - & - & 0.50\end{array}$

Modal SDC's, $(\%)$

$\begin{array}{lcccc}\psi_{1} & 0.5 i 1 & 2.60 & 2.58 & 2.78 \\ \psi_{2} & 5.566 & 3.90 & 2.41 & 3.87 \\ \psi_{3} & 3.426 & 2.74 & 2.71 & 2.81 \\ \psi_{4} & 0.656 & 0.82 & 0.82 & 0.63 \\ \dot{\psi}_{5} & 4.540 & 2.22 & 2.20 & 2.18 \\ \text { Max. static deflection (tip), (in) } & 0.0199 & 0.0500 & 0.0500 & 0.0499\end{array}$

Modal amplitudes (tip), (in)

$\begin{array}{lllll}U_{z 1}^{d} & 0.934 & 1.536 & 1.534 & 1.518 \\ U_{z 2}^{d} & 0.000 & 0.000 & 0.000 & 0.000 \\ U_{z 3}^{d} & 0.000 & 0.244 & 0.243 & 0.240 \\ U_{z 4}^{d} & 0.145 & 0.000 & 0.000 & 0.000 \\ U_{z 5}^{d} & 0.000 & 0.091 & 0.091 & 0.08 i\end{array}$

Natural Frequencies, $(\mathrm{Hz})$

$f_{1}$

$f_{2}$

$f_{3}$

$f_{4}$

$f_{5}$

$\begin{array}{rrrr}383 . & 252 . & 252 . & 248 . \\ 1089 . & 1360 . & 1534 . & 1338 . \\ 1565 . & 1572 . & 1572 . & 1558 . \\ 2395 . & 2964 . & 2968 . & 3518 . \\ 3854 . & 5029 . & 5027 . & 5053 .\end{array}$


Table 3 Optimum design for Case 2.

\begin{tabular}{lcc} 
Property & Unidirectional & Optimum \\
\hline Ply Angles, degrees & & \\
$\theta_{1}$ & 0.00 & 31.24 \\
$\theta_{2}$ & 0.00 & 31.74 \\
$\theta_{3}$ & 0.00 & 30.02
\end{tabular}

Fiber volume ratios

$\begin{array}{lll}k_{f 1} & 0.50 & 0.53 \\ k_{f 2} & 0.50 & 0.52 \\ k_{f 3} & 0.50 & 0.52\end{array}$

Modal SDC's, (\%)

$\begin{array}{lll}\psi_{1} & 0.5 \pi 1 & 3.35 \\ \psi_{2} & 5.566 & 4.10 \\ \psi_{3} & 3.426 & 3.34 \\ \psi_{4} & 0.656 & 0.70 \\ \psi_{5} & 4.540 & 2.59 \\ \text { Max. Static deflection (tip), (in) } & 0.0199 & 0.0660\end{array}$

Modal amplitudes (tip), (in)

$\begin{array}{lll}U_{z 1}^{d} & 0.934 & 1.775 \\ U_{z 2}^{d} & 0.000 & 0.000 \\ U_{z 3}^{d} & 0.000 & 0.280 \\ U_{z 4}^{d} & 0.145 & 0.000 \\ U_{z 5}^{d} & 0.000 & 0.101\end{array}$

Natural Frequencies, $(\mathrm{Hz})$

$\begin{array}{ccc}f_{1} & 383 . & 220 . \\ f_{2} & 1089 . & 1275 . \\ f_{3} & 1565 . & 1383 . \\ f_{4} & 2395 . & 3274 . \\ f_{5} & 3854 . & 4488 .\end{array}$




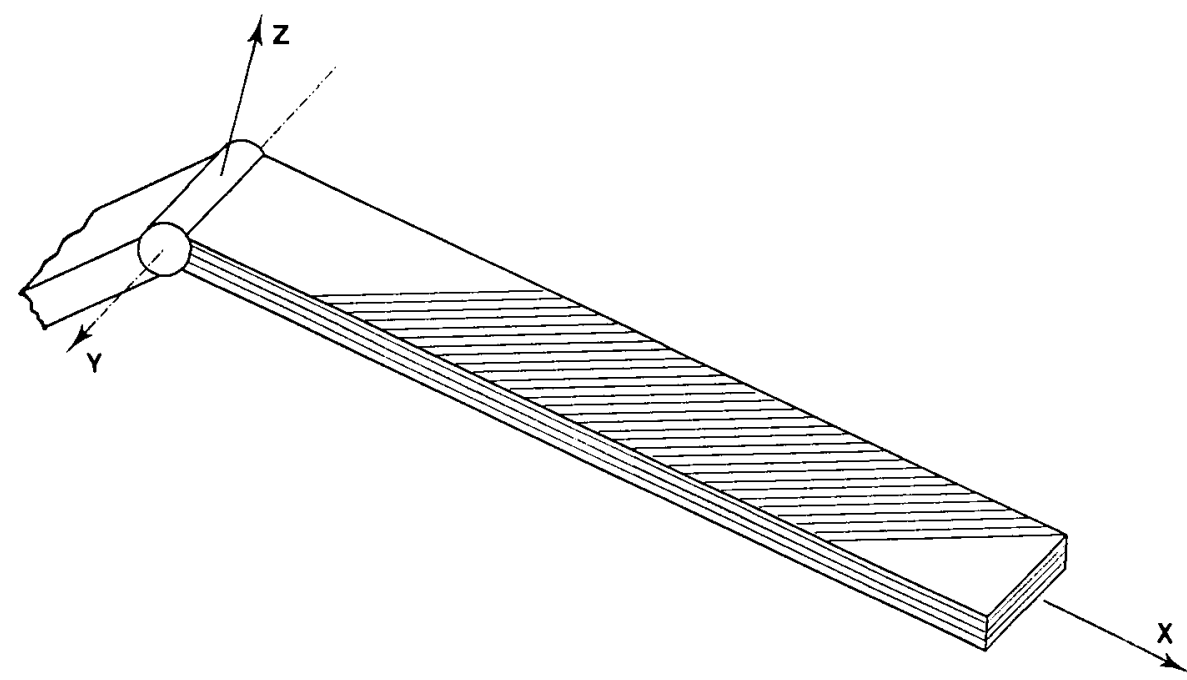

(A) LINK GEOMETRY.

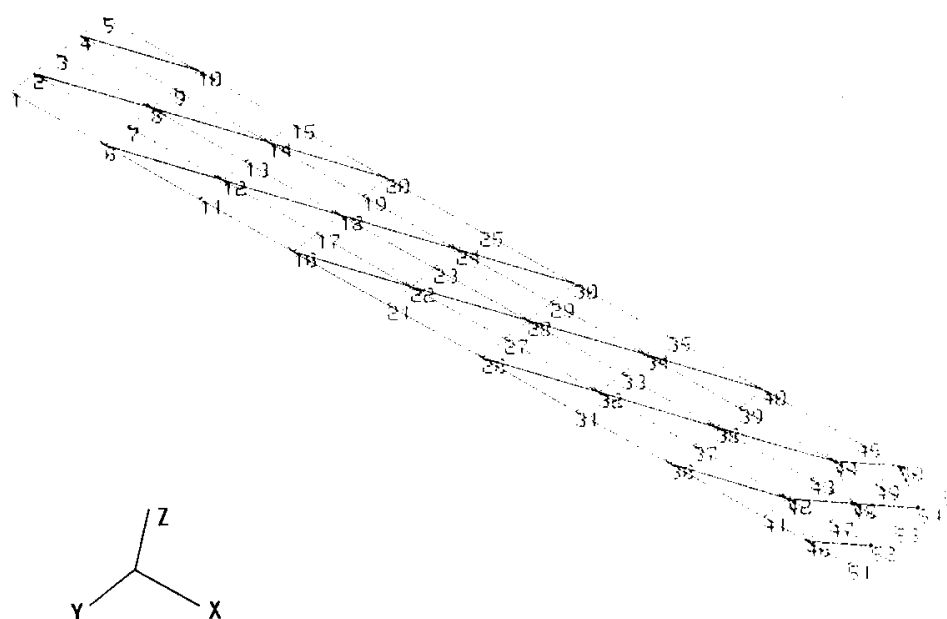

(B) FINITE ELEMENT MESH.

FIGURE 1. - TYPICAL COMPOSITE LINK.

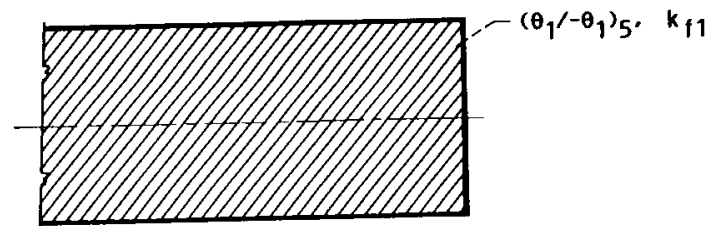

(A) ONE SUBLAMINATE.

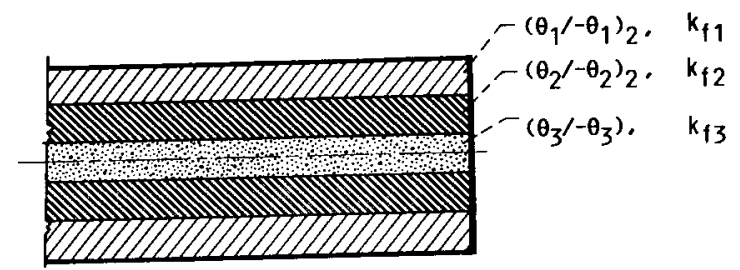

(B) THREE SUBLAMINATES.

FIGURE 2. - TYPICAL LAMINATE CONFIGURATIONS. 


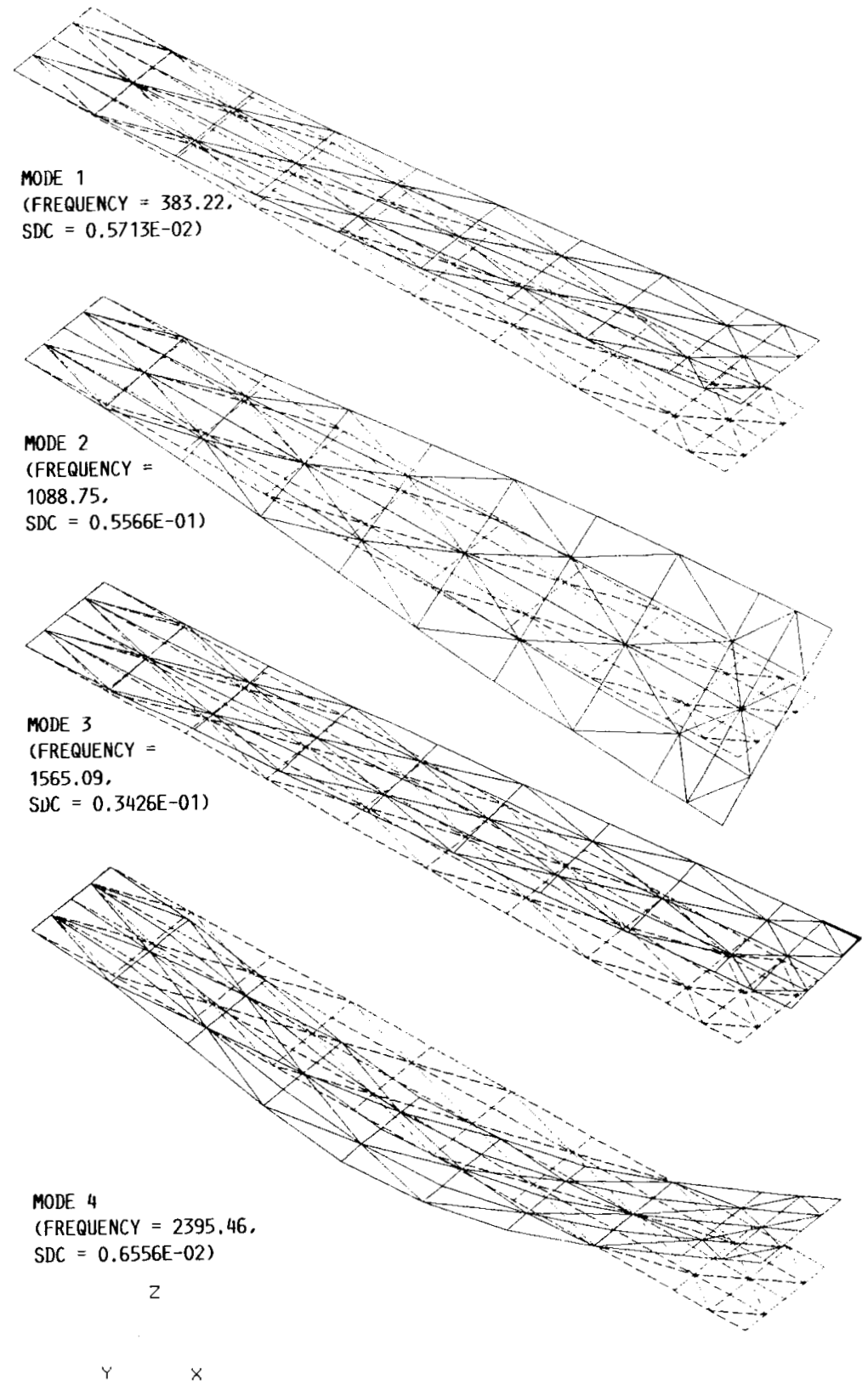

FIFURE 3. - MODE SHAPES OF THE UNIDIRECTIONAL COMPOSITE LINK. 


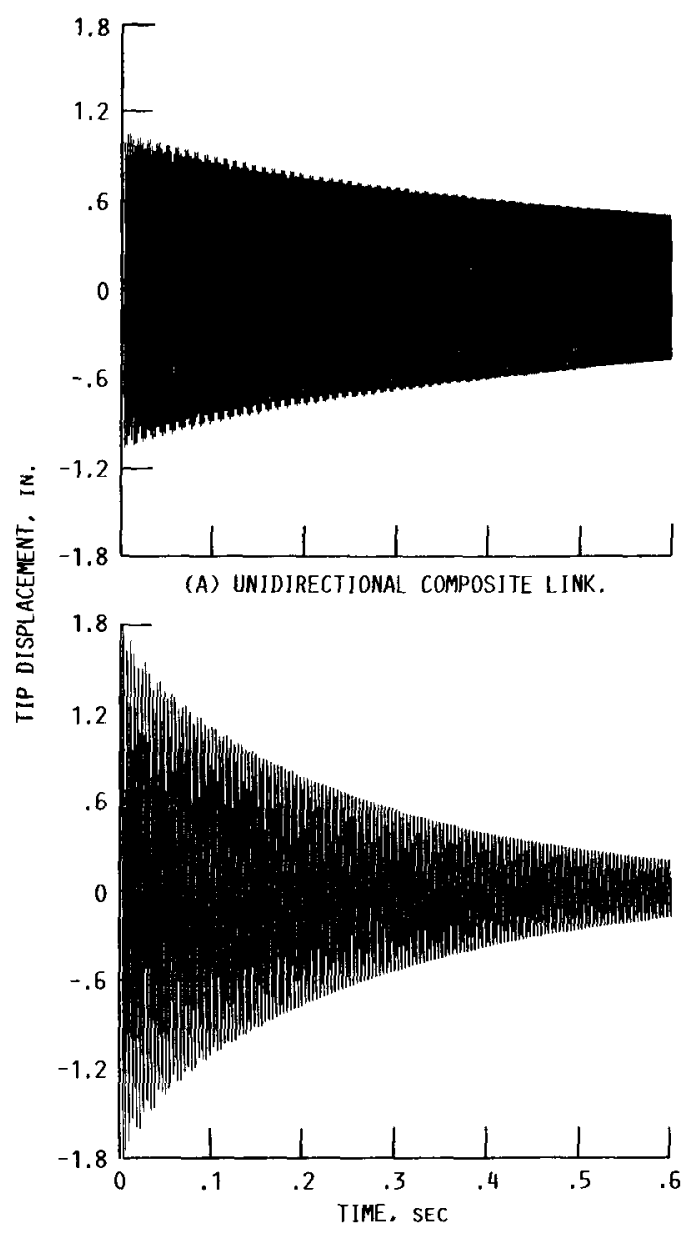

(B) OPTIMALLY TAILORED COMPOSITE LINK, CASE (1C).

FIGURE 5. - TRANSIENT IMPULSE RESPONSE. 


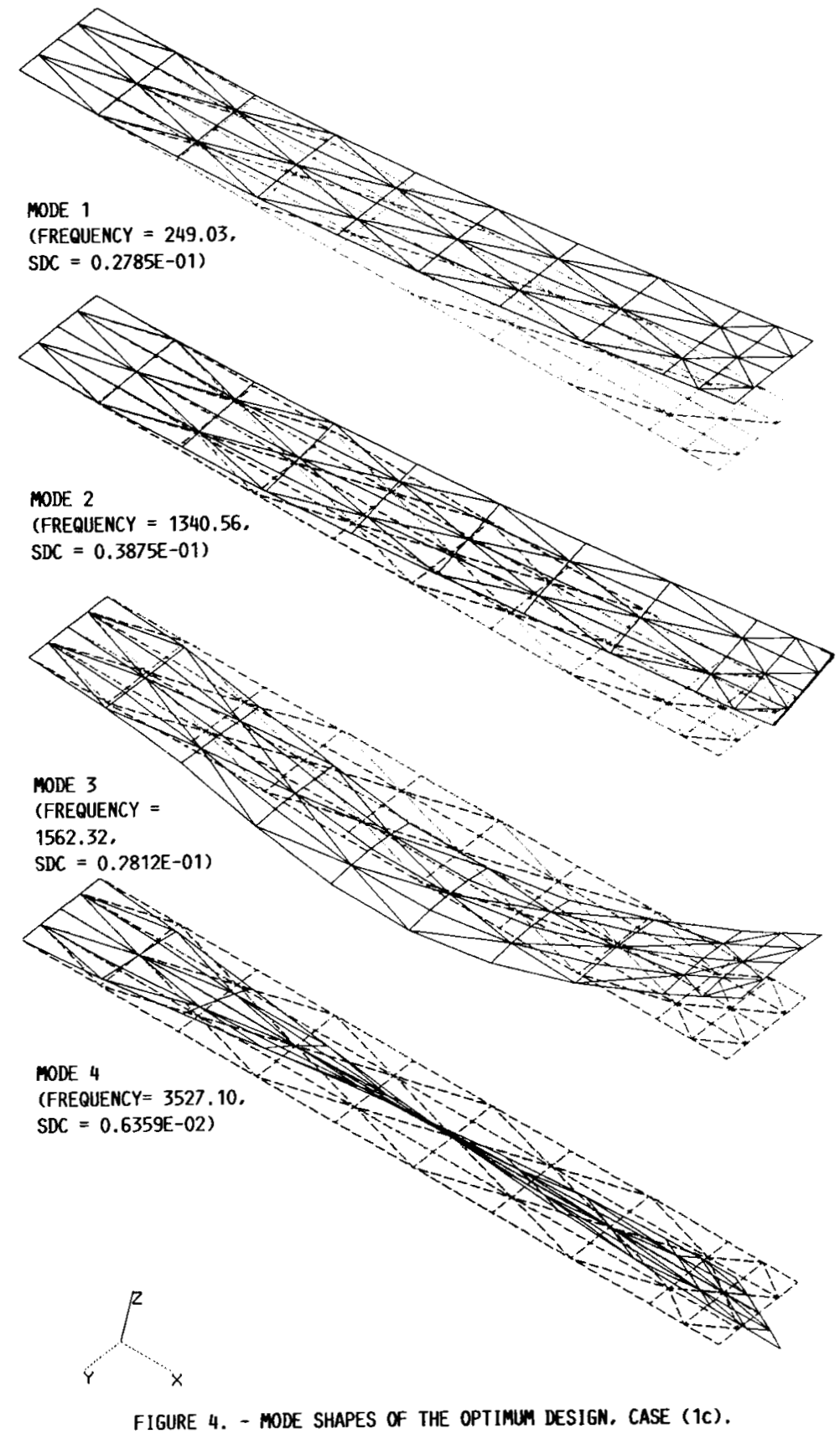




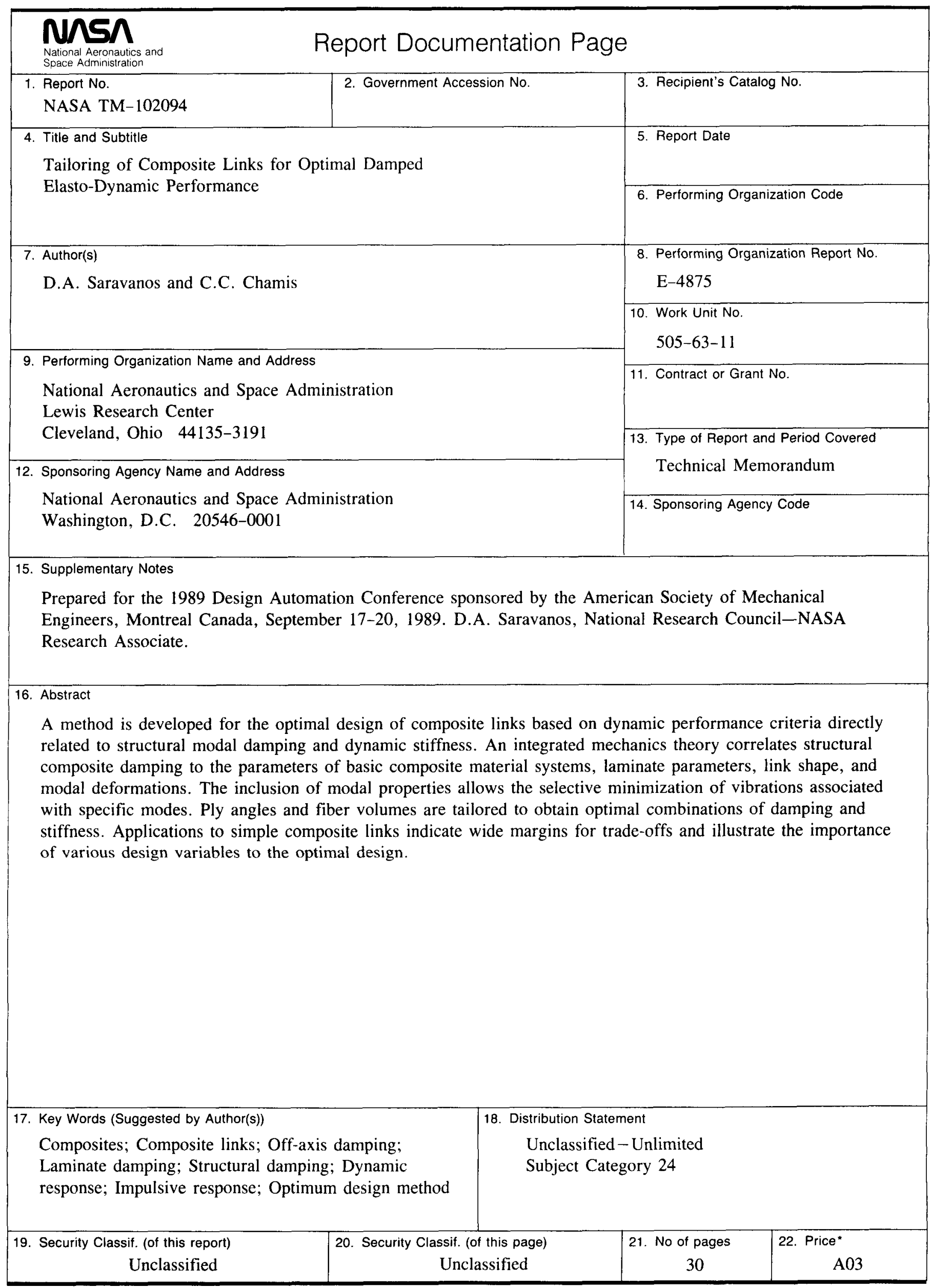

